\title{
SPATIAL CORRELATIONS OF DONOR CHARGES IN MBE CdTe*
}

\author{
T. Suski, P. Wiśniewski, E. Litwin-StaszewsKa \\ Unipress, Polish Academy of Sciences, Sokolowska 29, 01-142 Warszawa, Poland \\ D. Wasik, J. Przybytek, M. BaJ \\ Institute of Experimental Physics, Warsaw University \\ Hoża 69, 00-681 Warszawa, Poland
}

\author{
G. Karczewski, T. Wojtowicz, A. Zakrzewski and J. Kossut \\ Institute of Physics, Polish Academy of Sciences \\ Al. Lotników 32/46, 02-668 Warszawa, Poland
}

\begin{abstract}
We present experimental evidence that at high pressures indium donors in $\mathrm{CdTe}$ localize electrons in spatially correlated manner. We have studied Hall mobility, $\mu_{\mathrm{H}}$, as a function of electron concentration, $n_{\mathrm{H}}$, at $T=77 \mathrm{~K}$. Changes of $n_{\mathrm{H}}$ have been achieved by two methods. High pressure freeze-out of electrons onto localized states of In-donors leads to the mobility enhancement with respect to the situation when $n_{\mathrm{H}}$ has been modified by means of a subsequent annealing of the sample. As a result, depending on the degree of spatial correlations in the impurity charges arrangement, different values of $\mu_{\mathrm{H}}$ correspond to the same value of $n_{\mathrm{H}}$. The variation of mobility with electron concentration suggests that the localized state of In-donor represents likely negatively charged DX state.
\end{abstract}

PACS numbers: 71.55.Gs, 72.80.Ey

Indium donor in CdTe crystal introduces the localized level lying about $125 \mathrm{meV}$ above the bottom of the conduction band [1,2]. Application of hydrostatic pressure induces an upward shift of the localized level and of the conduction band ( $\mathrm{CB}$ ) minimum. Due to the higher value of the $\mathrm{CB}$ pressure coefficient the Fermi level approaches In related level and if temperature is not lower than about 90-100 K and at elevated pressures the impurities start to capture free electrons $[1,2]$. As a result the donor charge state changes. It has been established that the localized state of the donor has metastable properties and reveals some DX-like features. At temperatures lower than about $90 \mathrm{~K}$ barriers for electron capture and

*This work is supported by the State Committee for Scientific Research (Republic of Poland)under grants: 3P407 028 07, PBZ-101-01, and 8T11B 02108. 
emission on and from the localized state make the electron transfer between the impurity and the CB impossible.

It is well known that under a condition of a fractional occupancy of impurity states, it is possible for charges localized on the impurities to correlate their mutual positions (see e.g. [3]). As a consequence, the electron scattering by the charged centers has partially a coherent character. Two different mechanisms leading to the spatial correlations have been identified recently. The first one occurs in a system with impurities forming the positive and neutral charge states (positive- $U$ model). A distribution of these states among impurity sites may be sensitive to the Coulomb repulsive forces acting between positive charges localized on donors. They tend to occupy distant impurity sites. HgSe doped with Fe represents such a case $[3,4]$. The second type of a driving force which can lead to a correlated arrangement of impurity charges is realized in semiconductors with donors possessing a negative- $U$ character. Positively and negatively charged impurities tend to form dipole-like objects which results in a reduction of a scattering efficiency. GaAs and AlGaAs with donors (DX centers) illustrate the latter case [3]. The purpose of this work was to demonstrate experimentally the presence of the spatial correlation of In-donor charges in CdTe crystal. A possible mechanism leading to the appearance of these correlations was discussed also.

Indium doped $n$-CdTe layer $3 \mu \mathrm{m}$ thick was grown by molecular beam epitaxy at about $250^{\circ} \mathrm{C}$ on $\mathrm{GaAs}$ substrate with a $\mathrm{ZnTe}$ buffer. We have studied the population and depopulation processes of In-DX states located randomly among the CdTe lattice cation sites together with the relevant electron mobility changes. Variation of $\mu_{\mathrm{H}}$ with $n_{\mathrm{H}}$ were measured at atmospheric pressure. The pressure was used as a tool to obtain various nonequilibrium occupation of DX centers by means of high pressure freeze-out (HPFO) procedure. HPFO procedure consists of applying pressures exceeding about $0.75 \mathrm{GPa}$ at room temperature. It results in an enhanced occupation of DX centers which are in thermal equilibrium with the $n_{\mathrm{H}}$ at this temperature. Then, cooling of the sample at constant pressure occurs and this is followed by releasing of the pressure at $77 \mathrm{~K}$. The whole procedure results in a persistent lowering of $n_{\mathrm{H}}$ and its value decreases with increase in a freeze-out pressure.

An assumption about a presence, in the examined CdTe crystal, of the correlations in the arrangement of In-donor charges implies the situation when such pressure-induced capture of electrons onto DX centers favors high values of mobility corresponding to a given $n_{\mathrm{H}}$. It has been argued [5] that processes of electron capture on DX centers are sensitive to the fluctuations of a potential in a crystal. Electrons populate likely $d^{+}$centers located close one to another and depending on the mechanism which induces correlations, either more and more distant $d^{+}$ states or pairs of positively and negatively charged donor states, $d^{+}-\mathrm{DX}^{-}$, are formed. The method leading to the reduction of the correlation degree consists in a thermal ionization of DX centers. This treatment of the sample was employed after the reservoir of metastable electrons captured on DX centers was "prepared" by HPFO procedure. We have employed cycles of the sample annealing at temperatures exceeding roughly $90 \mathrm{~K}$. Every annealing cycle consisted of temperature increase from $77 \mathrm{~K}$, at which all measurements of $\mu_{\mathrm{H}}$ vs. $n_{\mathrm{H}}$ were performed. 
Higher annealing temperature causes more effective emission of electrons to the conduction band due to emptying of DX centers as they tend to their thermal equilibrium. The electron emission induced by annealing cycles occurs from randomly chosen DX state and therefore leads to the destruction of correlations in the distribution of charges among impurity sites [4].

To prove whether the above assumption about the correlated arrangement of impurity charges is correct we should demonstrate that higher values of $\mu_{\mathrm{H}}$ correspond to the $n_{\mathrm{H}}$ variation induced by HPFO procedure. The annealing should lead to lower $\mu_{\mathrm{H}}$ values. The obtained experimental results confirm this assumption. Solid circles in Fig. 1 correspond to a variation of $\mu_{\mathrm{H}}$ with decreasing $n_{\mathrm{H}}$

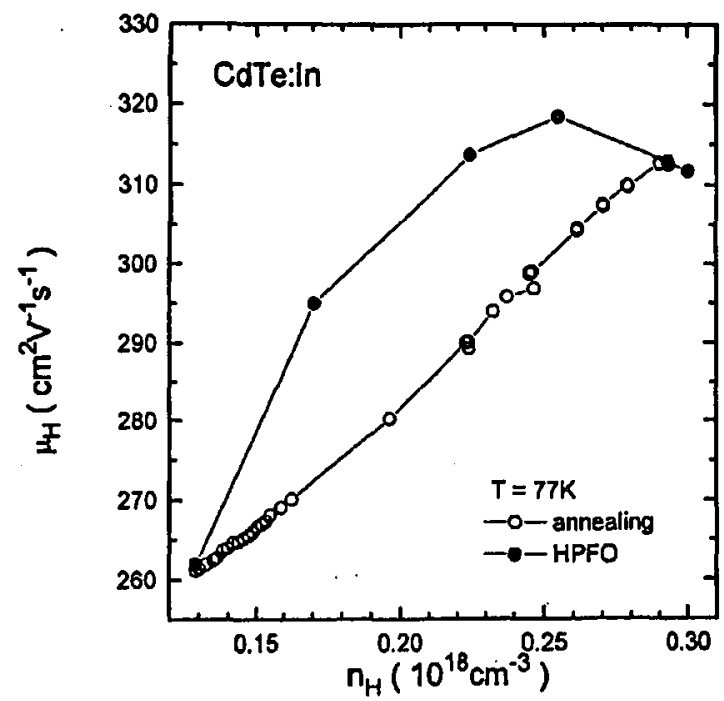

Fig. 1. Mobility of electrons as a function of Hall electron concentration measured at $T=77 \mathrm{~K}$ and ambient pressure. Solid circles represent the high pressure freeze-out which results in the high mobility trace. Open circles mark the lower mobility "path" which was achieved after subsequent annealing steps.

by means of HPFO procedure. The lower branch of $\mu_{\mathrm{H}}$ versus $n_{\mathrm{H}}$ in Fig. 1 (open circles) shows the path obtained during $n_{\mathrm{H}}$ increase by means of the annealing.

Now the problem of the mechanism leading to correlations will be analyzed. For this purpose we illuminated the sample (by a light dose from an infrared diode) subjected to the pressure of $1.2 \mathrm{GPa} . \mu_{\mathrm{H}}$ increases from 190 to $250 \mathrm{~cm}^{2} \mathrm{~V}^{-1} \mathrm{~s}^{-1}$. The corresponding persistent increase in $n_{\mathrm{H}}$ measured at $77 \mathrm{~K}$ was from about $1 \times 10^{17} \mathrm{~cm}^{-3}$ to $3 \times 10^{17} \mathrm{~cm}^{-3}$. Assumption about the positive- $U$ model for In-DX center should result in a weak decrease in the mobility with increasing $n_{\mathrm{H}}$. Mobility decrease induced by a conversion of neutral donors to positively charged scattering centers is compensated by efficient screening effect (higher density of conducting electrons). A small, positive contribution to the mobility due to correlations in arrangement of charges vanishes. Independently of the model correlations 
are destroyed by light. The negative- $U$ model gives a constant number of impurity charges. Illumination causes a conversion from negatively to positively charged donor states. Thus, the transfer of electrons to the CB gives more effective screening contribution to the scattering. This should lead to the increase in $\mu_{\mathrm{H}}$. The latter result is in agreement with the experimental observation. The above discussion was performed under the assumption that the compensation in the studied sample is small. If the opposite is true, the ratio of charged impurities depopulated by electrons during the sample illumination is low in comparison with all charged impurities present in the sample. Then, mobility changes predicted by the both models (positive- or negative- $U$ character of In-DX center) become similar.

Concluding, we have verified effects of spatial correlation of In-donor charges in CdTe crystal. An increase in the electron mobility with increasing $n_{\mathrm{H}}$ suggests that the model of negative- $U$ center is more appropriate for the localized state of In donor.

\section{References}

[1] G.W. Iseler, J.A. Kafalas, A.J. Strauss, Solid State Commun. 10, 619 (1972).

[2] D. Wasik, J. Przybytek, M. Baj, G. Karczewski, T. Wojtowicz, A. Zakrzewski, J. Kossut, Proc. European Workshop on II-VI Semiconductors, Linz 1994.

[3] J. Kossut, Z. Wilamowski, T. Dietl, K. Świątek, Proc. 20th Int. Conf. on the Physics of Semiconductors, Eds. E.M. Anastassakis, J.D. Joannopoulos, World Scientific, Singapore 1990 , p. 613.

[4] J. Mycielski, Solid State Commun. 60, 165 (1986).

[5] W. Jantsch, Z. Wilamowski, G. Ostermayer, Phys. Scr. Vol. T 45, 140 (1992). 